\section{Estimando o impacto da renda na saúde através de programas de transferência de renda aos idosos de baixa renda no Brasil}

\author{
Estimating the impact of income on \\ health through income transfer programs \\ for poor elderly in Brazil
}

Marcelo C. Neri 1,2

Wagner Lopes Soares 3,4

\footnotetext{
${ }^{1}$ Centro de Políticas Sociais, Fundação Getúlio Vargas,

Rio de Janeiro, Brasil.

2 Escola de Pós-graduação em Economia, Fundação Getúlio Vargas, Rio de Janeiro, Brasil. ${ }^{3}$ Instituto Brasileiro de Geografia e Estatística, Rio de Janeiro, Brasil.

4 Escola Nacional de Saúde Pública Sergio Arouca, Fundação Oswaldo Cruz, Rio de Janeiro, Brasil.

Correspondência M. C. Neri

Centro de Políticas Sociais, Fundação Getúlio Vargas. Praia de Botafogo 190, sala 801, Rio de Janeiro, $R J$ 22253-900, Brasil. mcneri@fgv.br
}

\begin{abstract}
Income levels and health status are related variables, because an increase in income enables greater access to assets that improve individual health status, and because productivity and income gains are associated with improved workers' health. Recent Brazilian experience offers favorable conditions to overcome the simultaneity problem that occurs when estimating the impact of income on health. A special health edition from both the 1998 and 2003 National Household Surveys (PNAD) shows the relationship between changes in these two variables. In addition, the Brazilian government has adopted and expanded several income transfer programs targeting the poor elderly population. The strategy used to identify the effect of income on health consisted of comparing the health status of eligible and non-eligible groups, before and after the expansion of income transfer programs. We used differences in estimates based on logistic regression. Our results show a distinct gain in the health status of lower-income seniors and do not allow rejecting the hypothesis that income changes have an impact on perceived health conditions.
\end{abstract}

Aged; Poverty; Income; Equity in Health
Introdução

Ao longo das últimas décadas o Brasil tem enfrentado mudanças significativas na sua estrutura etária, tendo em vista as transformações ocorridas no processo de envelhecimento populacional, o que significa um aumento relativo da parcela dos idosos, que tanto pode se dar via reduções na fecundidade ou/e na mortalidade nas idades mais avançadas. De acordo com Carvalho \& Garcia 1, essa mudança tem a sua resposta mais representativa no declínio da fecundidade e não invariavelmente na mortalidade, como imagina o senso comum. Aumentos de $300 \%$ da população idosa são esperados em países em desenvolvimento nos próximos anos. Um outro ponto observado no país é o incremento da longevidade, que representa o número de anos, em média, que um indivíduo, ou sua respectiva geração, viverá. Segundo dados divulgados pelo Instituto Brasileiro de Geografia e Estatística (IBGE), a esperança de vida ao nascer da população brasileira chegou a 71,2 anos de vida em 2000, contra os 62,2 anos alcançados em 1980 2. Um indivíduo com sessenta anos no ano 2000 esperaria viver em média mais 21,1 anos, e, nos anos 80 , uma pessoa com esta mesma idade esperaria viver em media mais 17,7 anos. Em síntese, esses dois eventos, concorrem tanto para aumentos relativos como também absolutos na população idosa brasileira. 
Em geral, essas mudanças foram direta, ou indiretamente, afetadas por políticas sociais adotadas no passado e de forma mais determinante nos campos da saúde pública e de transferência de renda. Dentre as principais políticas, destaca-se a universalização da seguridade social e do sistema público de saúde, adotada na Constituição Federal de 1988, que desvinculou o título meritório antes restrito apenas à condição de contribuinte, estendendo o seguro, a assistência social e o acesso aos serviços de saúde a outra parcela da população, antes não contemplada. São muitos os trabalhos que apontam os impactos sócio-econômicos das mudanças de transferências de renda no grupo dos idosos, após o novo texto constitucional 3,4. A maioria se refere às mudanças sobre a renda no subgrupo de maior idade, uma vez que esta passou a configurar uma parcela mais representativa dos rendimentos da população brasileira.

Além da Constituição Federal de 1988, outras políticas sociais mais recentes, especificamente entre 1998 e 2003, promoveram mudanças na vida dos brasileiros da terceira idade. Dentre essas políticas, destacam-se: a redução, em 1998, da idade mínima de recebimento do Beneficio de Prestação Continuada (BPC) 5, de 70 para 67 anos, e, recentemente, para 65 anos; a Política Nacional de Saúde do Idoso, em 1999, que promove medidas preventivas e atendimento multidisciplinar, com uma maior participação ativa do Programa Saúde da Família (PSF); o Estatuto do Idoso, em 2003, que estabelece direitos sociais e promove eqüidade em diferentes campos entre idosos e os demais membros da população, lhes conferindo cidadania e auto-estima; além da política de reajuste dos benefícios a partir de 1998, que passou a diferenciar os reajustes, privilegiando aqueles com valor igual ao piso constitucional.

Diferentemente do período pós-constituinte, não são muitos os trabalhos que avaliam os impactos dessas recentes políticas sob as condições de vida e na saúde no subgrupo dos idosos, em particular nos de baixa renda. A Pesquisa Nacional por Amostra de Domicílios (PNAD) de $1998{ }^{6} \mathrm{e}$ 2003 7, permite captar impactos dessas políticas, servindo como um instrumento de avaliação, uma vez que trazem um suplemento especial, permitindo avaliar e comparar as necessidades e o uso dos serviços de saúde. Para ser mais claro, seus dados permitem avaliar o antes e o depois dos efeitos de expansão e/ou de criação de alguns dos novos programas supracitados.

Por meio de um estimador de diferenças em diferenças baseado em regressões logísticas, este artigo estuda a relação entre renda e saúde, tendo como principal instrumento a análise dos impactos de políticas de transferência de renda para idosos de baixa renda no Brasil. Como grande parte do tempo vivido pelos idosos se dá sob as piores situações de saúde, um objetivo mais específico, mas não menos importante, é estudar políticas de renda como um dos determinantes dos indicadores de saúde dos idosos brasileiros, lançando mão de condições privilegiadas para contornar o problema de endogeneidade na estimativa do impacto da renda na saúde, em função da disponibilidade de dados para os períodos pré e pós-implementação de políticas de transferência de renda.

\section{Materiais e métodos}

Os dados foram extraídos de suplemento especial de pesquisas domiciliares do IBGE (PNAD $1998{ }^{6}$ e 2003 7), e fornecem condições inéditas no caso brasileiro para avaliar o impacto de alterações da renda através de mudanças nos critérios de acesso a programas de transferência de renda sobre três indicadores de saúde: necessidades de cuidados de saúde; acesso e o consumo de serviços de saúde.

Dentre as variáveis da PNAD que indicam necessidade dos cuidados de saúde foram escolhidas duas: "auto-avaliação do estado de saúde: bom ou muito bom" e "esteve acamado nas duas últimas semanas anteriores à pesquisa". Evitamos utilizar variáveis de morbidade autoreferida, tendo em vista que estes dados podem subestimar as prevalências de doenças ou condições crônicas, em razão de problemas de memória e/ou ausência de diagnóstico 8,9. O acesso aos serviços de saúde (privado) foi comparado por meio da posse do seguro de saúde ("possui plano de saúde"), ao passo que a procura por serviços médicos hospitalares foi avaliada por intermédio da variável "procurou por serviços de saúde nas duas semanas anteriores à pesquisa”.

No presente artigo, procurou-se dar mais ênfase à variável auto-avaliação da saúde do que aos aspectos sobre a capacidade funcional, muito embora a literatura aponte esta última como muito significante para avaliação das condições de vida dos idosos. A escolha se pautou pelo fato de a auto-avaliação da saúde apontar para características que extrapolam o significado de saúde no sentido estrito, e ganha representatividade quanto ao bem-estar do indivíduo. Embora não represente um exame quanto à presença física ou mental de doenças, o entrevistado, quando auto-avaliar o seu estado de saúde, leva em conta inúmeras variáveis consideradas subjetivas (ansiedade, mal-estar, autocontrole, vitalidade). Por essa razão, a hipótese de observar os resultados como uma escala ajustada de bem-estar 
geral não é muito forte, tendo em vista os componentes que cada indivíduo, de maneira diferente, ponderam ao se auto-avaliarem.

O artigo é composto de duas etapas metodológicas. Inicialmente, se compara, entre 1998 e 2003, a proporção das variáveis selecionadas, segundo a idade dos indivíduos. Por exemplo, confronta-se a proporção de indivíduos com 61 a 65 anos de idade, que auto-avaliaram a saúde boa ou muito boa, em 1998, com a obtida em 2003. Nesse caso, um descolamento das curvas de probabilidades pode sinalizar indiretamente mudanças no padrão de comportamento do "nível de bem-estar" em determinado grupo etário, o que pode sugerir efeitos das políticas já mencionadas, caso se dê no grupo dos idosos.

Uma maneira mais dinâmica de avaliação é considerar os mesmos eventos em uma análise de coorte de nascimento. A limitação desse tipo de análise é que os dados de coortes de nascimento são substitutos imperfeitos de dados longitudinais, uma vez que não fornecem informações sobre os mesmos indivíduos ao longo do tempo. $\mathrm{Na}$ verdade, as informações são de diferentes indivíduos com um certo conjunto de características idênticas, no caso época de nascimento.

Diferentemente da comparação de proporções de uma determinada faixa etária entre 1998 e 2003, a análise de coorte permite avaliar se houve alterações na probabilidade de ocorrência desses eventos entre indivíduos de uma mesma geração. Isso significa comparar proporções de indivíduos que, por exemplo, reportaram boa saúde em 1998, quando tinham de 56 a 60 anos, com um número de pessoas com este mesmo desfecho em 2003, quando possuíam de 61 a 65 anos de idade. Dessa forma, reduções em proporções sugerem globalmente uma melhoria (espontânea ou no tratamento) das afecções ou a redução do número de casos novos, sendo que, o contrário, indica um aumento de casos novos ou um baixo número de reversões do problema. Ambos os resultados estariam diretamente relacionados às medidas de caráter preventivos e curativos, refletindo, de certa forma, os efeitos de algumas medidas adotadas.

Na segunda etapa, estimamos a diferença em diferença dos indicadores de saúde entre idosos de baixa renda e os demais, entre 1998 e 2003. O grupo de tratamento é composto por aqueles elegíveis a programas de transferência de renda, ou seja, aqueles com mais de 65 anos e renda domiciliar per capita inferior a $1 \frac{2}{2}$ salário mínimo - sem contar a influência dos benefícios sociais. Esses dois grupos antes e depois da aplicação de novas políticas de transferência de renda são a base do experimento aqui em questão. São controlados por dummy de ano, a dummy que representa os indivíduos idosos de baixa renda e a interação destas duas variáveis dummies, que como veremos, nos fornece o estimador de diferença em diferença entre os elegíveis e aqueles não-elegíveis das mudanças proporcionadas sobre saúde nos pontos extremos do período em questão.

Matematicamente, podemos representar o método de diferenças em diferenças com a seguinte equação:

g3 $=\left(¥_{98}\right.$, idade $>65 \_<1 / 2$ salário mínimo $-¥_{03}$, idade $>65 \_<1 / 2$ salário mínimo $)-\left(\Psi_{98}\right.$, idade $<65$ > 1/2 salário mínimo $-¥_{03}$, idade $<65$ _ $1 / 2$ salário mínimo)

Onde cada $¥$ representa a proporção da variável estudada para cada ano e grupo, com o número subscrito representando o período da amostra (1998, para antes da mudança, e 2003, para depois da mudança) e a letra representando o grupo ao qual o dado pertence (idade $<65$ _ $1 / 2$ salário mínimo para o grupo de controle, e idade $>65 \_<1 / 2$ salário mínimo para o grupo de tratamento). E g3 será nossa estimativa a partir das diferenças em diferenças. Obtendo g3 determinamos o impacto do experimento natural sobre a variável que gostaríamos de explicar.

Representando o método através de uma regressão e criando as variáveis indicadoras (ou dummies): $\mathrm{dB}$, igual a um para os indivíduos do grupo de tratamento (idade $>65 \_<1 / 2$ salário mínimo) e zero para os indivíduos do grupo de controle (idade $<65$ _ 1/2 salário mínimo); e d2, igual a um quando os dados se referem ao segundo período, pós-mudança (2003), e zero caso os dados se refiram ao período pré-mudança (1998); temos:

$\mathrm{Y}=\mathrm{g} 0+\mathrm{g} 1^{*} \mathrm{~d} 2+\mathrm{g} 2{ }^{*} \mathrm{~dB}+\mathrm{g} 3^{*} \mathrm{~d} 2 * \mathrm{~dB}+$ outros fatores

Onde Y representa a variável estudada, por exemplo, "auto-avaliação do estado de saúde: bom ou muito bom", gl o impacto de estar em 2003 sobre a variável estudada, g2 o impacto de estar no grupo de tratamento sobre a variável estudada, e g3 o impacto pós-evento do grupo de controle sobre a variável endógena escolhida. Assim, g0 capta justamente o valor esperado da variável estudada quando se analisa o grupo de controle antes da mudança, o que nos dá, basicamente, o parâmetro de comparação.

Além do mais, é interessante controlar por outros fatores relevantes na regressão, o que significa dizer que, antes de alegarmos que g3 nos dará o impacto da política exógena sobre os indicadores de saúde selecionados, temos de descobrir e isolar o efeito de todas as outras variáveis que podem causar mudanças na variável de desfecho, o que é feito inserindo algumas variáveis de controle relevantes na regressão: acesso à água, esgoto, sexo, idade, idade ao quadrado, 
cor (brancos e não-brancos), educação (0, 1-4, 4-8, 8-12, mais de 12 anos de estudo), migração (migrantes e não-migrantes), situação (rural e urbana) e Unidades da Federação.

\section{Resultados}

\section{Mudanças entre grupos etários e por coorte}

Comparamos proporções de eventos relacionados à saúde, entre 1998 e 2003, e avaliamos a ocorrência de mudanças nas suas respectivas distribuições etárias. Adicionalmente à análise das mudanças ocorridas na mesma faixa etária entre dois pontos no tempo, ligamos os que representam uma coorte, ou seja, traçamos uma linha entre a proporção obtida em 1998 em uma determinada faixa etária e a encontrada em 2003 na faixa etária subseqüente. Por exemplo, ligamos a proporção de pessoas com "auto-avaliação do estado de saúde: bom ou muito bom” de 56 a 60 anos em 1998 com aquela encontrada no subgrupo populacional de 60 a 64, em 2003. Isso significa avaliar o quanto, em média, a necessidade dos serviços de saúde alterou, ao longo do ciclo de vida dessa geração no período considerado. Uma inclinação positiva (negativa) entre as duas proporções indica aumento (decréscimo), respectivamente, na variável avaliada entre os dois períodos estudados.

As figuras trazem essas informações segundo faixa etária para três indicadores de saúde: necessidades de uso de serviços de saúde (autoavaliação, e esteve acamado); acesso a plano de saúde e procurou serviços de saúde.

Na Figura 1, inicialmente, observa-se uma queda significativa nas proporções de indivíduos que reportaram auto-avaliação do estado de saúde bom ou muito bom à medida que se caminha ao longo da distribuição de idade, o que sugere o efeito natural do processo de envelhecimento, que está associado ao acúmulo de problemas crônicos degenerativos. Outro ponto é que se observa um pequeno descolamento das duas curvas a partir dos cinqüenta anos de idade, com queda mais lenta no ano de 2003, o que sugere uma melhoria da trajetória do ciclo da vida do nível de bem-estar em geral para essas faixas etárias. Apesar da melhoria, verifica-se, na analise de coorte, que os indivíduos com idade mais avançada e de uma mesma geração, tendem, em geral, a declarar uma avaliação menos otimista a respeito do seu estado de saúde, uma vez que se observam inclinações negativas nos traços que representam as coortes, o que reflete o efeito do acúmulo dos anos de vida sobre a saúde dos indivíduos. Por exemplo, verifica-se que cerca de $45 \%$ dos indivíduos com 61 a 65 anos consideravam o seu estado de saúde bom ou muito bom em 1998, ao passo que, em 2003, dentre aqueles com 66 a 70 anos de idade, esse numero chega a $44 \%$. O ponto central é que em 1998 na população nessa mesma faixa etária de 66 a 70 anos a proporção de avaliações positivas de saúde era de $40 \%$.

Quanto à variável "esteve acamado nas duas últimas semanas anteriores à pesquisa”, verificase que nas faixas etárias que compreendem 21 a 40 anos de idade não se observam diferenças nas proporções de indivíduos com este desfecho em 1998 e 2003. Entretanto, em 2003, verifica-se uma queda na proporção de acamados para as faixas etárias mais avançadas, fato não ocorrido entre os mais jovens. Como fruto da redução do número de indivíduos acamados nas idades mais avançadas, essa proporção também diminui ao longo da trajetória de uma mesma geração. Nota-se que daqueles que tinham entre 61 e 65 em 1998, 7,42\% estavam acamados nas duas últimas semanas da pesquisa, mas chegam, em 2003, quando tinham de 66 a 70 anos de idade, com um contingente inferior, 7,02\%. Essa mesma faixa etária em 1998 apresentava uma taxa de $8,75 \%$. Isso sugere eficácia de alguns procedimentos de reabilitação, ou mesmo a redução de novos casos. O mesmo já não acontece com a última coorte, pela impossibilidade de realizar esse tipo de analise, pois a última faixa etária é aberta agregando indivíduos com mais de setenta anos, ou seja, mais de uma geração.

A variável de procura efetiva por serviços de saúde nas últimas duas semanas demonstrada no Figura 2, apresenta incrementos de um comportamento mais errático, em particular nas idades mais avançadas. $\mathrm{O}$ maior incremento se dá na proporção dos indivíduos que em 1998 tinham de 61 a 65 anos de idade que passa de $20,2 \%$ para $23,9 \%$ em 2003. Entretanto, não é possível diferenciar a influência neste padrão de elementos de demanda de saúde de alguma forma relacionados ao quesito relativo a se a pessoa esteve acamada no mesmo período de 15 dias e elementos de oferta. Procuramos contornar esse problema analisando a seguir uma variável relacionada à oferta de serviços privados de saúde.

No que diz respeito ao acesso a seguro saúde, observa-se um comportamento mais errático das mudanças das distribuições etárias com aumento na proporção de indivíduos com plano de saúde nos extratos e idade mais avançada. A proporção de acesso a plano de saúde nas faixas etárias com idade mais avançada que 55 anos é maior em 2003, ao passo que entre os indivíduos mais jovens este fato em geral não é verdade. Na geração dos indivíduos que tinham de 61 a 64 anos em 1998, a posse de seguro saúde sofre 
Figura 1

Variáveis de saúde, segundo faixas etárias e coortes de nascimento, 1998 e 2003.

1a) Considera seu estado de saúde bom ou muito bom

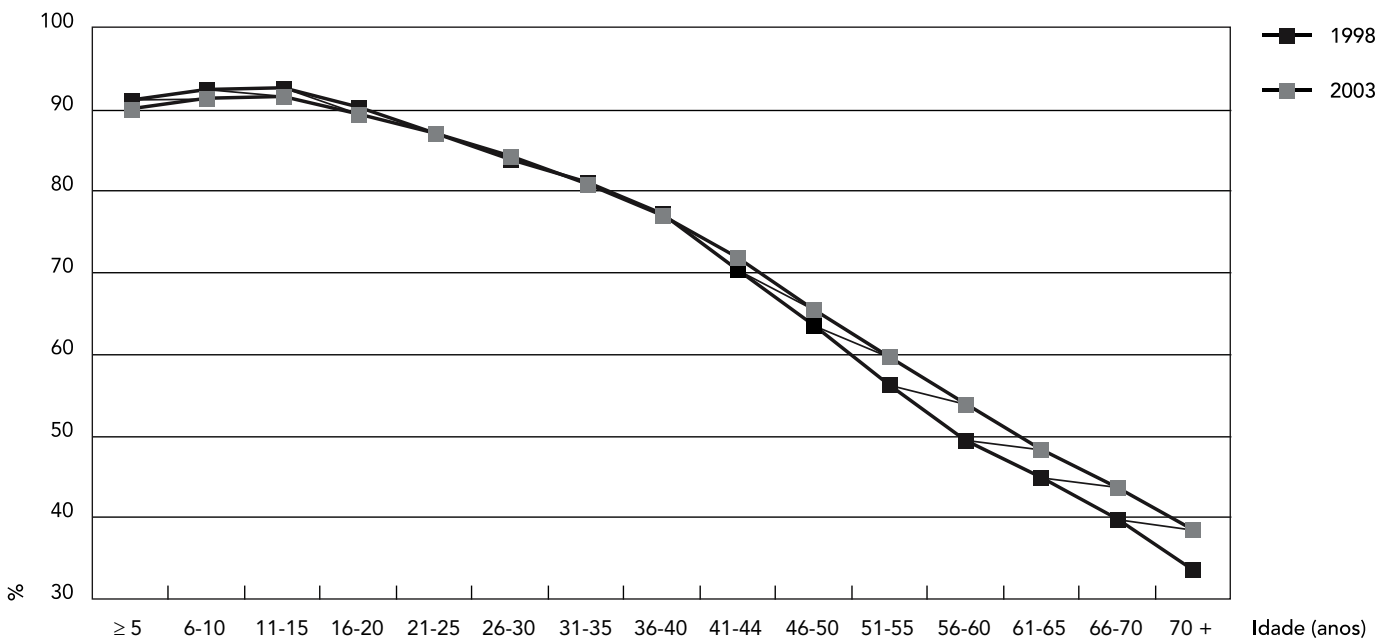

1b) Esteve acamado na duas últimas semanas

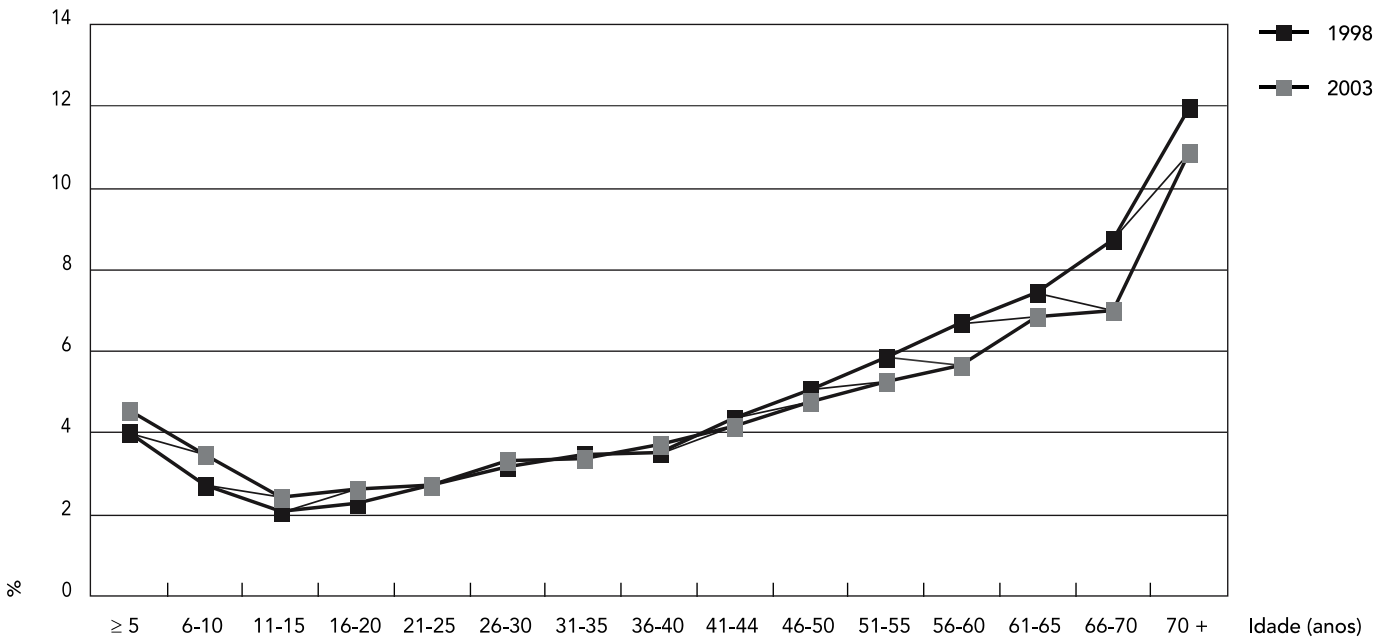

(continua)

um incremento de $27,7 \%$ para $29,4 \%$ no período analisado. Esse crescimento no acesso a planos privados de saúde é consistente com ganhos relativos de renda dos mais velhos e do aumento na procura de serviços de saúde observado num contexto de melhoria dos indicadores sanitários.

\section{Análise bivariada}

A pesquisa demonstra que de todos os grupos etários, os idosos com 65 anos ou mais de idade foram os que obtiveram melhoria mais expressiva dos indicadores de saúde no período de 1998 
1c) Procurou serviço de saúde nas duas últimas semanas

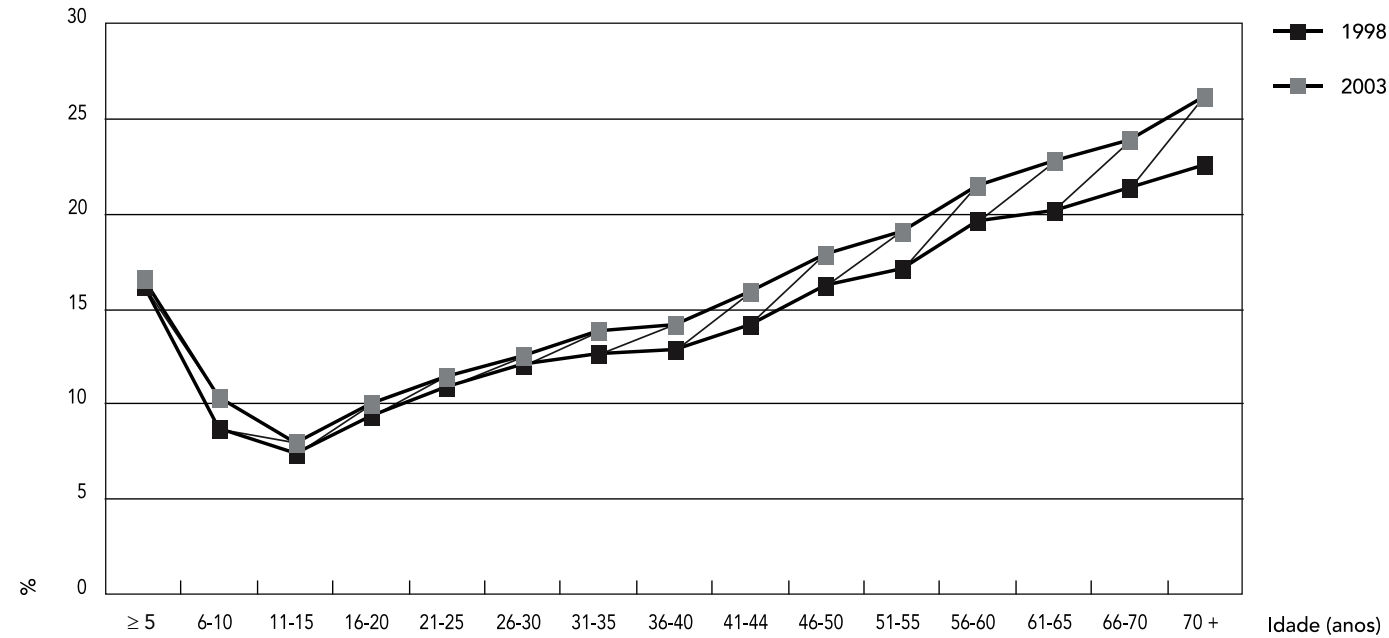

1d) Tem direito a algum plano de saúde

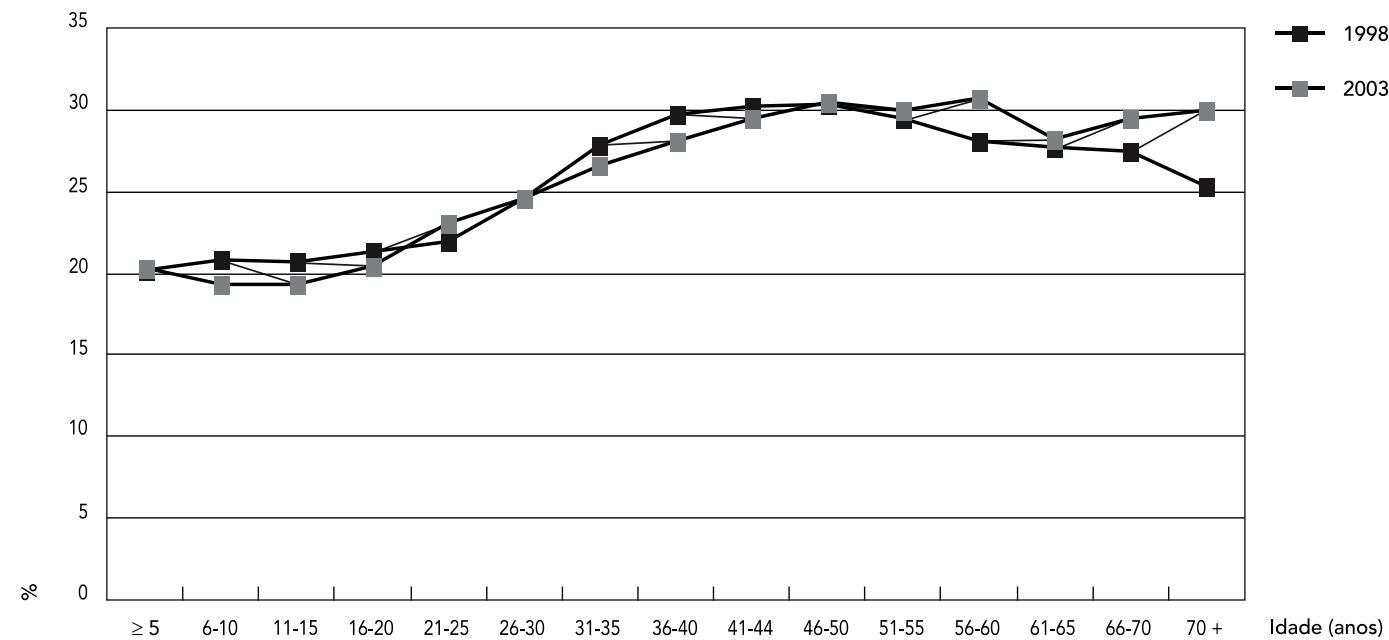

a 2003. Podemos sintetizar esses resultados através de algumas estatísticas resumo bi-variadas, a saber: (i) a proporção de idosos que auto-avaliavam o seu estado de saúde como bom ou muito bom sobe de $36,5 \%$ para $40,8 \%$, enquanto a de não-idosos passou de $81,8 \%$ para $81,3 \%$; (ii) a proporção de idosos que ficaram acamados nos últimos 15 dias cai de $10,4 \%$ para $9,3 \%$, enquanto a de não-idosos quase não se alterou, passando de $3,52 \%$ para $3,7 \%$ e; (iii) a proporção de idosos com acesso a planos privados de saúde sobe de $26,1 \%$ para $29,7 \%$, enquanto mais uma vez a de não-idosos ficou mais ou menos estável (de $24,3 \%$ para $24,2 \%$ ).

A proporção de idosos que procuraram serviços de saúde nos últimos 15 dias sobe de $26,1 \%$ 
para $29,7 \%$, enquanto a de não-idosos sobe de $12,4 \%$ para $13,8 \%$. Esse elemento é ambíguo, pois depende tanto da oferta de serviços como da demanda, função da condição de saúde da pessoa. Como esse aumento da procura de serviços de saúde acontece num contexto de melhoria dos indicadores de saúde e do acesso a planos privados de saúde, ele é consistente com ganhos relativos de renda dos mais velhos que serão agora detalhados na análise de diferença em diferença.

\section{Diferenças em diferenças}

A Tabela 1 traz os resultados das razões de chance controladas e não-controladas de "esteve acamado nas duas últimas semanas anteriores à pesquisa" e "auto-avaliação do estado de saúde: bom ou muito bom". Nesse caso, apresentamos as razões de chances controladas ou não para as variáveis: ano (2003), idosos com renda menor que $1 / 2$ salário mínimo (grupo de tratamento); e a interação entre essas duas variáveis, o que sugere o estimador de diferença em diferença que capta os efeitos das políticas no período sobre o grupo de tratamento. As saídas completas das regressões, com os coeficientes estimados para as demais variáveis controles, podem ser obtidas em Neri \& Soares 8 , mas, de maneira geral, os resultados são consistentes com os encontrados na literatura, sendo o melhor estado de saúde encontrado em homens, nativos, mais educados, que moram em áreas rurais e com acesso a esgoto.

Observa-se que quando controlamos as outras variáveis, a dummy idosos > 65_renda domiciliar per capita $<1 / 2$ salário mínimo reduz as chances de estar acamado, o que já não acontece quando não controlamos os efeitos das outras variáveis relevantes ou de confusão. Nesse caso, pessoas com mais de 65 e renda inferior a $1 / 2$ salário mínimo têm $5 \%$ a menos de chance de estar acamado, ou seja, o acumulo de mais idade entre os mais vulneráveis, com características sócio-econômicas semelhantes, não representa um aumento deste evento, pelo contrário, representa uma queda, embora não muito expressiva.

Já uma queda mais expressiva, cerca de 18\%, pode ser observada quando avaliamos as chances obtidas pelo estimador de diferença em diferença, ou melhor, a interação entre idoso com mais de 65 anos com renda familiar inferior a $1 / 2$ salário mínimo e a variável ano de 2003, resultado que fornece indiretamente o efeito específico das políticas de saúde e renda durante o período analisado, especificamente para o subgrupo populacional elegível aos benefícios sociais. Por outro lado, quando avalia-se apenas o efeito de mudança de período sem considerar um determinado grupo etário, tanto no experimento controlado quanto no não-controlado, verifica-se aumentos nas chances de ficar acamado, o que sugere uma piora global no período neste indicador para a população em geral.

Quando se avalia o estado de saúde bom ou muito bom, verifica-se que ser idoso com mais de 65 anos e renda per capita inferior a 1/2 salário mínimo possui chances reduzidas de $89 \%$ e $8 \%$ de reportar estado de saúde bom ou muito bom, quando o experimento é controlado ou não, respectivamente. Tal fato evidencia o papel do acumulo dos anos de idade no processo de saúde das populações.

A Tabela 2 apresenta as razões de chance controladas ou não-controladas de variáveis de acesso à saúde, como o "possui plano de saúde", e de

Tabela 1

Razão de chance: variável endógena "esteve acamado nas duas últimas semanas anteriores à pesquisa" e "auto-avaliação do estado de saúde: bom ou muito bom".

\begin{tabular}{|c|c|c|c|c|}
\hline \multirow[t]{2}{*}{ Variáveis } & \multicolumn{2}{|c|}{$\begin{array}{l}\text { "Esteve acamado nas duas últimas } \\
\text { semanas anteriores à pesquisa" }\end{array}$} & \multicolumn{2}{|c|}{$\begin{array}{l}\text { "Auto-avaliação do estado de saúde: } \\
\text { bom ou muito bom" }\end{array}$} \\
\hline & Não-controlado & Controlado * & Não-controlado & Controlado * \\
\hline Idoso > 65_renda domiciliar & 3,099 & $0,949 * *$ & 0,111 & 0,918 \\
\hline \multicolumn{5}{|c|}{ per capita $<1 / 2$ salário mínimo } \\
\hline Ano de 2003 & 1,075 & 1,079 & 0,937 & 0,904 \\
\hline Interação entre as variáveis & 0,845 & 0,816 & 1,170 & 1,330 \\
\hline
\end{tabular}

* Controles: acesso à água, esgoto, sexo, cor (brancos e não-brancos), educação (0, 1-4, 4-8, 8-12, mais de 12 anos de estudo), migração (migrantes e não-migrantes) e Unidades da Federação;

** Estatisticamente não-significante em nível de 95\%.

Fonte: Centro de Políticas Sociais, Fundação Getúlio Vargas, Rio de Janeiro, Brasil. 
Tabela 2

Razão de chance: variável endógena "possui plano de saúde" e "procurou por serviços de saúde nas duas últimas semanas anteriores à pesquisa".

\begin{tabular}{|c|c|c|c|c|}
\hline \multirow[t]{2}{*}{ Variáveis } & \multicolumn{2}{|c|}{ "Possui plano de saúde" } & \multicolumn{2}{|c|}{$\begin{array}{l}\text { "Procurou por serviços de saúde nas duas } \\
\text { últimas semanas anteriores à pesquisa" }\end{array}$} \\
\hline & Não-controlado & Controlado * & Não-controlado & Controlado * \\
\hline \multicolumn{5}{|l|}{ Idoso > 65_renda domiciliar } \\
\hline per capita $<1 / 2$ salário mínimo & 0,715 & 0,569 & 1,849 & 0,795 \\
\hline Ano de 2003 & 0,959 & 0,641 & 1,117 & 1,080 \\
\hline Interação entre as variáveis & 0,730 & 1,328 & $0,971 \star \star$ & $1,033 * \star$ \\
\hline
\end{tabular}

demanda como "procurou por serviços de saúde nas duas últimas semanas anteriores à pesquisa". Quando comparamos a evolução entre os anos, há redução em $35 \%$ nas chances controladas de acesso a seguro de saúde (reduz em $4 \%$ quando não-controlado). Os idosos com renda de até 1/2 salário mínimo são aqueles com as menores chances de acesso (menos $43 \%$ e 18,5\%, controlado e não-controlado, respectivamente). Agora quando analisamos esse mesmo grupo numa perspectiva temporal, observamos ganho relativo quando controlado por outras características (aumento de 32\%). O mesmo não ocorre no exercício não-controlado. Esse resultado evidencia um impacto positivo sobre o acesso a plano de saúde no subgrupo específico de idosos de baixa renda no período, quando comparado com o grupo sem essa característica.

Quando se avalia a procura por serviços de saúde, verifica-se que ser idoso com mais de 65 anos e renda per capita inferior a $1 / 2$ salário mínimo possui chances reduzidas de $21,5 \%$, quando controlamos por outras variáveis relevantes. Por outro lado, quando se avalia o impacto da mudança de período analisado (1998 para 2003) nesse grupo especifico (de tratamento) se observa aumento na procura por serviços de saúde.

Deve-se ressaltar que como não se pode observar a distribuição de renda dentro dos domicílios e o processo de cadastramento junto a programas sociais, dever-se-ia trabalhar com dois conceitos polares de renda, a saber: a renda domiciliar per capita que corresponde ao caso de socialização perfeita da renda do idoso e dos demais membros no interior do domicílio e o caso de renda individual do idoso. Nesse caso, os mesmos exercícios foram realizados, mas considerando a renda individual e não a renda domiciliar per capita inferior a 1⁄2 salário mínimo. Como resultado, não foram observadas mudanças significativas nas estimativas.

\section{Conclusão}

Lima-Costa et al. 9 baseados na PNAD, concluem que idosos com renda mais baixa apresentam piores condições de saúde, pior função física e um menor uso dos serviços de saúde. Entretanto, esses indicadores quando avaliados ao longo do tempo apresentam uma melhoria relativa especificamente nesse subgrupo populacional. Coincidentemente ou não, o mesmo aconteceu com a renda dos idosos no período. A renda per capita de famílias com idosos foi entre as famílias de todos os grupos etários a que mais aumentou entre 1991 e 2003, sendo que, no grupo de indivíduos com sessenta anos ou mais, a renda sofreu um incremento real de $43 \%$, e para os indivíduos de 20 a 29 anos de idade, por exemplo, a variação foi de $19,3 \%$.

Dados da Pesquisa de Orçamentos Familiares (POF) 10 de 2003 apontam que os idosos possuem na opinião dos próprios membros de suas respectivas famílias, um nível de vida melhor que as demais faixas etárias na maioria dos aspectos estudados. Cerca de 13\% das pessoas com mais de sessenta anos de idade admitiram que a quantidade de alimentos normalmente consumidos pela família não é suficiente, ao passo que no grupo de menor idade, como o 10 a 19 anos, este número chega a 19\%. Quando o assunto é condições de moradia, 55\% dos indivíduos com mais de sessenta anos consideram boas as condições do seu domicílio, enquanto que entre os indivíduos de 10 a 19 anos de idade chega a $45 \%$. 
De certo, o trabalho tem sido apontado como um determinante para uma maior sobrevida e o desemprego tem sido associado a uma pior condição de saúde, mortalidade, problemas psiquiátricos ${ }^{11}$. Por outro lado, melhorias nas condições de saúde promovem também maior sobrevida no mundo do trabalho, ou seja, idosos saudáveis têm maior chance de trabalhar em idades mais avançadas. Esse é exatamente o problema de medir e avaliar impactos sobre a saúde via ganhos de rendimentos obtidos pelo mercado de trabalho, ou seja, a existência de causalidade inversa ou endogeneidade entre a renda e a saúde. Giatti \& Barreto 12 mostraram que a saúde funcional, que indica uma maior autonomia e mobilidade física, é um importante preditor para a permanência na vida ativa dos idosos. Baseados na PNAD 1998 6, verificam que mais de $1 / 4$ dos idosos das regiões metropolitanas brasileiras trabalhavam, número considerado expressivo quando comparado com outros países. Segundo os autores, embora exista aposentadoria compulsória para os mais idosos, o Brasil não segue a tendência mundial, pois, de maneira peculiar, o sistema previdenciário não requer que o aposentado se afaste da vida laboral, estimulando a sua permanência no mundo do trabalho.

Entretanto, no Brasil a grande parcela da renda dos idosos não é obtida via trabalho; as transferências governamentais e as ações assistenciais são fundamentais, tornando-se um importante braço para mudanças nas condições de saúde da população idosa. Portanto, a experiência brasileira de políticas desse tipo acaba abrindo espaço para a análise de avaliação do impacto da renda sobre a saúde. Nesse caso, a diferença de avaliação está no fato do incremento de renda se dar via programas de transferência e assistenciais, o que de certa forma contorna o problema de endogeneidade do seu efeito sobre a saúde.

Em linhas gerais, quando se analisa a evolução etária de indicadores gerais de saúde, como, por exemplo, a auto-avaliação subjetiva do estado de saúde e a freqüência que a pessoa ficou acamada, entre outras, nota-se melhorias absolutas nas faixas etárias mais avançadas, sendo que quando acompanhamos a trajetória destes indicadores por gerações, observa-se uma maior estabilidade dos indicadores gerais de saúde usados para as coortes acima dos sessenta anos, quando esperar-se-ia um decréscimo fruto do processo de envelhecimento. O resultado mais geral do artigo é a existência de um ganho diferenciado das condições de saúde da população com idade avançada e baixa renda, o que é consistente com a existência de um efeito-renda com a causalidade emanando desta em direção aos indicadores de saúde.
Em termos de políticas públicas, esses exercícios sugerem um impacto dos novos programas nas variáveis de saúde dos indivíduos mais velhos: o grupo dos idosos diminuiu a sua necessidade de cuidados de saúde, aumentando a proporção e as chances dos indivíduos se autoavaliarem como pelo menos bom o seu estado de saúde e diminuindo as daqueles que estiveram acamados nas duas últimas semanas. De certo, não só a renda foi um dos canais para melhoria do estado de saúde dos idosos de baixa renda, mas também a Política Nacional de Saúde do Idoso, que trouxe resultados no campo da assistência e prevenção à saúde, como, por exemplo, a vacina contra influenza e o advento da Farmácia do Idoso. Deve-se salientar que o indicador de percepção da saúde tem se mostrado robusto para avaliar o estado de saúde dos idosos porque prediz de forma consistente a sobrevida dessa população 9,13 .

Do lado das transferências de renda, segundo Camarano \& Pasinato 14, a redução do limite de idade para elegibilidade do BPC em 1998 aumentou em 253\% o número de beneficiários entre 1997 e 1999. Se tomarmos o período de 1997 a 2003 como um todo, o número de BPC para idosos sobe $648 \%$. Se considerarmos de maneira conjunta o BPC e a Renda Mensal Vitalícia para Idosos, esta última um programa em redução, o número de benefícios vigentes sobe $72,9 \%$ entre 1997 e 2003. Além do aumento do número de benefícios assistenciais houve um aumento real do salário mínimo deflacionado pelo Índice Nacional de Preços ao Consumidor (INPC), que corresponde ao piso dos benefícios previdenciários de 22,3\% no período de 1997 a 2003. Quando computamos a evolução do valor real da massa de todos os benefícios previdenciários, observamos um incremento de 44,4\% no período de 1997 a 2003. Como a política de reajustes dos benefícios previdenciários passou a partir de 1998 a diferenciar os reajustes dos benefícios iguais ao piso constitucional, entre outros voltados para os demais, e a criação de novos benefícios como o BPC visou às rendas domiciliares per capita inferiores a um quarto de salário mínimo, o efeito do aumento das transferências de renda sobre os idosos de renda mais baixa foi ampliado.

Outros resultados bastante relevantes foram as melhorias no acesso a plano de saúde na população idosa de menor renda e o aumento na procura de serviços de saúde. Em parte, o primeiro ponto pode ser explicado pelos incrementos dos planos básicos de saúde, ligados a categorias profissionais e planos populares, e aos mecanismos de redução de diferenciação de preços por idade do Estatuto do Idoso, muito embora este se encontre no limiar do período considerado. De- 
ve-se ressaltar que mesmo assim os seus efeitos de curto prazo não podem ser totalmente desprezados.

O fato é que com ou sem necessidade a pessoa pode consumir serviços de saúde, e a posse do seguro saúde pode induzir o uso desnecessário dos serviços (exames, procedimentos médicos, consultas, internações). Talvez isso possa sugerir o aumento observado na proporção e nas chances de procurar serviços de saúde no grupo de idosos em 2003, uma vez que se verificou uma redução da necessidade dos cuidados de saúde nesta faixa etária no mesmo ano. Outro ponto é que o consumo efetivo de serviços de saúde não necessariamente se traduz na demanda por saúde, uma vez que existem fatores que restringem o seu acesso (distância, períodos de espera, tempo, custo privado do tratamento).

Resumindo, entre 1998 e 2003, o grupo de idosos de menor renda, sujeito aos aumentos exógenos de transferências, reduziu a sua necessidade de cuidados médicos hospitalares, mas aumentou a procura por serviços de saúde, o que pode ser reflexo do maior acesso a planos de saúde por parte dessa população e algumas medidas no bojo da Política Nacional de Saúde ao Idoso, que melhoram o acesso aos serviços, como por exemplo, a expansão do PSF. Verificase que idosos têm maior comprometimento com as suas capacidades funcionais, o que impede em muito o deslocamento para atendimento em uma unidade hospitalar, ambulatorial ou consultório, tornando muito importante o papel do atendimento domiciliar, mais especificamente do médico de família.
Além dos ganhos sobre a saúde dessa população, as políticas de transferência têm alterado o papel do idoso dentro da família, lhe dando maior autonomia, isto é, desconstruindo o velho estigma de dependentes, tendo em visa que muitos familiares encontram-se dependentes da renda do idoso e compartilham do seu acúmulo patrimonial. Os idosos de hoje, pelo contrário, têm se revelado os principais provedores da renda familiar, situação que lhes confere maior valorização dentro da família e da sociedade em geral. Segundo Camarano et al. 14, o aumento das taxas de chefia entre idosos e a redução na proporção que reside na casa de parentes, sugerem uma diminuição na dependência dos idosos.

Em suma, os resultados sugerem um impacto indireto de novos programas de transferência de renda nas variáveis de saúde, mas não fazem menção à relação custo/benefício destes vis à vis à de outros programas públicos como de saneamento, educação e até mesmo de saúde. Em relação a esse último, este artigo, além de enfatizar o papel das políticas de transferência de renda, aponta para a importância de investimentos diretos na saúde da população com idade mais avançada, uma vez que os idosos, além de possuírem maior prevalência de cuidados médicos em relação aos demais indivíduos da população, apresentam intervenções médicas mais custosas e de tecnologia mais complexa, tendo em vista que, em geral, as doenças na terceira idade são crônicas e múltiplas, exigindo acompanhamento médico e medicação contínua 15. Um diagnóstico precoce e medidas preventivas reduziriam os custos dos procedimentos, o que de certa forma advoga a Política Nacional de Saúde do Idoso. 


\section{Resumo}

Nível de renda e estado de saúde são variáveis correlacionadas tanto pelo fato de aumentos da primeira propiciarem maior acesso a bens e serviços, que se refletem em melhorias no estado de saúde das pessoas, como pelos ganhos de produtividade e de renda propiciados por melhorias da saúde do trabalhador. Este artigo estuda os impactos da renda na saúde no Brasil, tendo como instrumento para lidar com o problema de simultaneidade as mudanças observadas em políticas de transferência de renda aos idosos de baixa renda. A estratégia usada foi comparar o estado de saúde de pessoas idosas de baixa renda-sem contar o efeito dos benefícios - antes e depois do incremento exógeno do recebimento de novos programas de transferência de renda. Utilizamos um estimador de diferenças em diferenças, baseado em regressões logísticas sobre suplementos especiais de saúde da Pesquisa Nacional por Amostra de Domicílios - PNAD 1998 e 2003. O trabalho demonstra uma melhora diferenciada do estado de saúde de pessoas idosas de baixa renda, o que não nos permite rejeitar a hipótese de que mudanças de renda causam melhorias nas condições percebidas de saúde.

Idoso; Pobreza; Renda; Eqüidade em Saúde

\section{Referências}

1. Carvalho JAM, Garcia RA. O envelhecimento da população brasileira: um enfoque demográfico. Cad Saúde Pública 2003; 19:725-33.

2. Instituto Brasileiro de Geografia e Estatística. Tábuas completas de mortalidade - 2005. http:// www.ibge.gov.br/home/estatistica/populacao/ tabuadevida/2005/default.shtm (acessado em 21/ Jun/2006).

3. Delgado G, Cardoso J. O idoso e a previdência rural no Brasil: a experiência recente da universalização. In: Castro AA, organizador. Novos idosos brasileiros. Muito além dos 60 ? Rio de Janeiro. Instituto de Pesquisa Econômica Aplicada; 2004. p. 293-319.

4. Beltrão K, Pinheiro S. A Constituição de 1988 e o acesso da população rural brasileira à segurança social. In: Castro AA, organizador. Novos idosos brasileiros. Muito além dos 60 ? Rio de Janeiro. Instituto de Pesquisa Econômica Aplicada; 2004. p. 321-51.

\section{Colaboradores}

M. C. Neri e W. L. Soares participaram igualmente de todas as etapas de elaboração do artigo.
5. Brasil. Lei no. 8742. Lei Orgânica da Assistência Social, de 07 de dezembro de 1993, dispõe sobre a organização da assistência social e dá outras providencias. Diário Oficial da União 1993; 8 dez.

6. Instituto Brasileiro de Geografia e Estatística. Pesquisa Nacional por Amostra de Domicílios - PNAD 1998. Rio de Janeiro: Instituto Brasileiro de Geografia e Estatística; 1998.

7. Instituto Brasileiro de Geografia e Estatística. Pesquisa Nacional por Amostra de Domicílios - PNAD 2003. Rio de Janeiro: Instituto Brasileiro de Geografia e Estatística; 2003.

8. Neri M, Soares W. Políticas sociais, renda e saúde na terceira idade. In: XV Encontro Nacional da Associação Brasileira de Estudos Populacionais. http://www.abep.nepo.unicamp.br/encontro2006/ docspdf/ABEP2006_720.pdf (acessado em 22/ Out/2006). 
9. Lima-Costa MF, Barreto SM, Giatti L. Condições de saúde, capacidade funcional, uso de serviços de saúde e gastos com medicamentos da população idosa brasileira: um estudo descritivo baseado na Pesquisa Nacional por Amostra de Domicílios. Cad Saúde Pública 2003; 19:735-43.

10. Instituto Brasileiro de Geografia e Estatística. Pesquisa de orçamentos familiares 2002-2003: microdados. Brasil e grandes regiões [CD-ROM]. Rio de Janeiro: Instituto Brasileiro de Geografia e Estatística; 2004.

11. Blanc PD, Katz P Yelin E. Mortality risk among elderly workers. Am J Ind Med 1994; 26:543-7.

12. Giatti L, Barreto SM. Saúde, trabalho e envelhecimento no Brasil. Cad Saúde Pública 2003; 19:75971.
13. Helmer C, Barberger-Gateau P, Letenneur L, Dartiques JF. Subjective health and mortality in French elderly women and men. J Gerontol B Psychol Sci Soc Sci 1999; 54:S84-92.

14. Camarano A, Pasionato M. O envelhecimento populacional na agenda das políticas publicas. In: Castro AA, organizador. Novos idosos brasileiros. Muito além dos 60? Rio de Janeiro. Instituto de Pesquisa Econômica Aplicada; 2004. p. 253-92.

15. Veras R. Em busca de uma assistência adequada à saúde do idoso: revisão da literatura e aplicação de um instrumento de detecção precoce e de previsibilidade de agravos. Cad Saúde Pública 2003; 19:705-15.

Recebido em 27/Abr/2006

Versão final reapresentada em 01/Fev/2007

Aprovado em 07/Fev/2007 